\title{
The Influence of Motion Control, Neutral and Cushioned Running Shoes on Foot Kinematics
}

\author{
Ben Langley ${ }^{a^{*}}$, Mary Cramp ${ }^{\mathrm{b}}$ and S. C. Morrison ${ }^{\mathrm{c}}$ \\ ${ }^{a}$ Edge Hill University, UK \\ bUniversity of the West of England, UK \\ ${ }^{\mathrm{c}}$ University of Brighton, UK
}

\section{Introduction}

Footwear biomechanics research typically focuses on the assessment of frontal plane rearfoot $(\mathrm{RF})$ motion when determining the influence of footwear on foot motion (Lilley et al., 2013; Cheung \& Ng, 2007). The key limitation of this approach is that it only describes one aspect of foot motion. Application of a multi-segmental foot model to the assessment of the shod foot could more accurately describe how footwear influences foot motion. There is currently limited exploration of the influence of running shoes on intersegmental foot kinematics due to the challenges of modelling the foot within the shoe.

\section{Purpose of the study}

To determine the influence of running shoes on foot kinematics.

\section{Methods}

Twenty-eight active males ( $26 \pm 7$ years, $1.77 \pm$ $0.05 \mathrm{~m}, 79 \pm 9 \mathrm{~kg})$ ran at a self-selected pace $(2.9$ $\pm 0.6 \mathrm{~m} / \mathrm{s}$ ) on a treadmill, in standardised ASICS motion control, neutral and cushioned running shoes. Kinematic data were collected using a VICON motion analysis system, operating at $200 \mathrm{~Hz}$. Ankle joint kinematics were calculated using a two segment model of the foot and shank. Intersegmental foot motion was calculated using the 3DFoot model (Leardini et al., 2007). Incisions were made within the shoe to enable direct tracking of the shod foot (Langley et al., 2015). Angles at initial contact (IC), peak angle and time to peak angle were extracted for analysis. One-way repeated measures ANOVA and Friedman's ANOVA were used to explore differences between conditions.

\section{Results}

There were significant increases in RF dorsiflexion upon IC ( $p=.01, W=.16)$ and peak dorsiflexion ( $p=.02, \mathrm{~W}=.14$ ) when running in the neutral shoe compared to the motion control and cushioned shoes. Peak RF eversion was significantly $(p=.04, W=.12)$ increased when running in the motion control shoe compared to the cushioned shoe. There was a significant increase in RF adduction upon IC $\left(p=.03, \eta^{2}=\right.$ $.12)$ and reduction in peak RF abduction ( $p=$ $.01, \eta^{2}=.15$ ) when running in the neutral shoe compared to the motion control shoe. There were significant reductions in MF-RF eversion upon IC $(p=.01, W=.16)$ and MLA deformation ( $p=.04, W=.11)$ when running in the motion control shoe compared to the neutral and cushioned shoes. No other significant differences were reported.

\section{Discussion and conclusion}

Motion control running shoes were shown to reduce aspects of intrinsic foot motion such as medial longitudinal arch deformation and MF- 
RF eversion (Figure 1B \& C). These findings were expected based on the design features of the shoe in comparison to the neutral and cushioned shoes. However, the motion control shoe did not reduce peak RF eversion (Figure 1A). This finding is in contrast to the previous literature (Lilley et al., 2013; Cheung \& Ng, 2007) and the design aims of the shoes. This disparity between the current study and the literature is likely due to this work assessing the motion of the foot within the shoe. The findings of this study suggest that motion control shoes were better at controlling intrinsic foot rather than RF motion. However, it should be noted that while statistically significant, both the magnitude of change $\left(\leq 3.32^{\circ}\right)$ and the effect sizes $(\leq .16)$ were small. The novel information developed within this study provides a more comprehensive understanding of how footwear influences foot motion which may aid in shoe development and potentially a reduction in running injury rates.

\section{References}

Cheung R, \& Ng, G. (2007) Phys Ther Sport, 8 (2), 75-81.

Langley, B. et al., (2015) J Foot Ankle res, 7 (S1), S144-45.

Leardini, A. et al., (2007) Gait Posture, 25 (3), 453-62.

Lilley, K. et al., (2023) Gait Posture, 37 (3) 331-5.
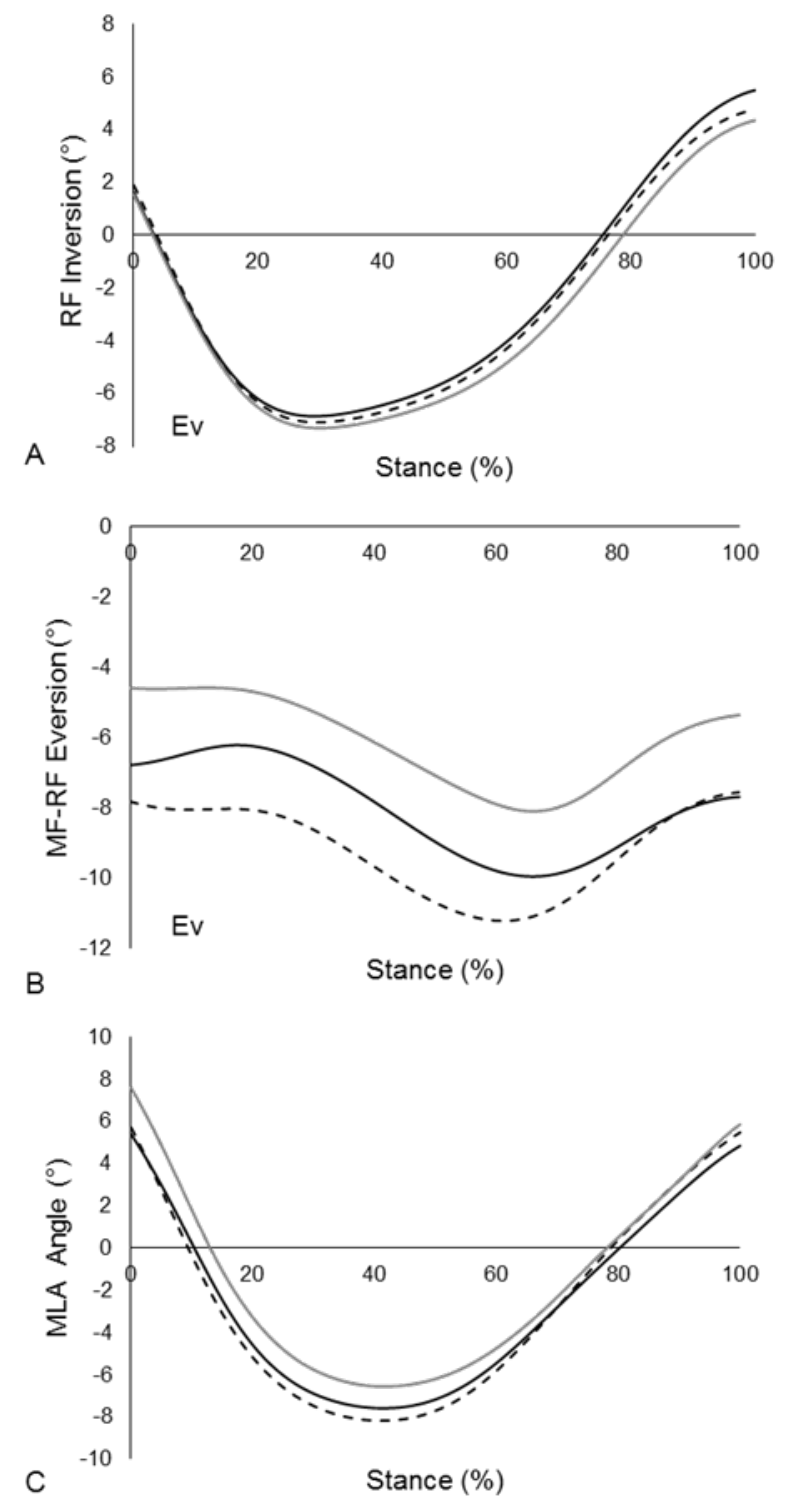

Figure 1. (A) Frontal plane rearfoot, (B) frontal plane midfoot to rearfoot and (C) medial longitudinal arch kinematics over the stance phase of running in motion control (solid grey line), neutral (solid black line) and cushioned (dashed black line) running shoes. Data averaged across all participants $(\mathrm{n}=28)$. 